\title{
A Method for Automatical Extraction of Typical Disaster-bearing Targets from LiDAR Point Cloud in Coastal Zone
}

\author{
Ping WANG \\ South China Sea Institute of Planning and Environmental \\ Research, SOA \\ Guangzhou, China \\ 1903125@qq.com \\ Yu-chao SUN \\ South China Sea Institute of Planning and Environmental \\ Research, SOA \\ Guangzhou, China \\ 503598481@qq.com \\ Fan YANG \\ South China Sea Institute of Planning and Environmental \\ Research, SOA \\ Guangzhou, China \\ 459078506@qq.com
}

\author{
Zheng WEI * \\ South China Sea Institute of Planning and Environmental \\ Research, SOA \\ Guangzhou, China \\ 155050074@qq.com, Corresponding author
}

Ji-sheng ZENG

South China Sea Institute of Planning and Environmental Research, SOA

Guangzhou, China

969735@qq.com

\section{Ling TANG}

South China Sea Institute of Planning and Environmental Research, SOA

Guangzhou, China 286744071@qq.com

\begin{abstract}
This paper presents a method based on georeferenced feature image to automatically extract typical disaster-bearing targets from coastal LiDAR data. Firstly, the noise and the water surface of LiDAR point cloud are removed by using the elevation histogram. Secondly, by analyzing the spatial distribution of point cloud, the georeferenced feature image of point cloud is generated. Finally, the image processing method and the corresponding relationship between the threedimensional point cloud and the two-dimensional feature image are used to realize the automatic extraction of the targets. In this paper, the LiDAR cloud data of Haidian Island acquired by ALS70, is used as experimental data to verify the feasibility and practicability of the proposed method.
\end{abstract}

Keywords-coastal zone;disaster bearing targets;LiDAR;object extraction

\section{INTRODUCTION}

In recent years, with the development of marine economy, a variety of coastal development activities are more and more, making the coastal zone is facing increasing pressure, resources and the environment has undergone unprecedented changes in marine disasters frequently. Such as the frequent typhoon storm surge in recent years has produced a great threat to the social security of coastal communities and watersheds, and caused great economic losses [1]. The detection, surveying and dynamic monitoring of the coastal disaster-bearing targets are the key to the integrated management, development and sustainable development of coastal zone. It is very important to study the automatic and dynamic monitoring methods of the disaster-bearing body in the coastal zone.

With the development of LiDAR technology, airborne LiDAR provides powerful technical support for fast, efficient and direct access to the three-dimensional information of the target. Many scholars use LiDAR point cloud data for coastal erosion, coastal zone extraction and other aspects of the study.

In terms of coastal erosion and shoreline extraction, the impact of storm surges on the coast of Sefton in the UK and the trend of future coastal zones are analyzed and calculated based on the sedimentation of airborne LiDAR point cloud [2]. Reference [3] proposed a method of automatically extracting the tidal reference coastline in airborne LiDAR point cloud based on image segmentation. The Monte Carlo method was used to analyze the influence of LiDAR vertical measurement error and tide datum uncertainty on the result of coastline extraction.

In the extraction of coastal targets, Reference [4] estimated and analyzed the changes of the targets such as land surface, coastline, vegetation cover and artificial building of Galveston Island, based on the airborne LiDAR data of Galveston East and West Bay acquired in 2010 and 2012. Pada et.al. (2016) used LiDAR in the mangrove extraction in the different coastal areas of Negros Occidental in Western Visayas, Philippines [5]. Derivatives of LiDAR data such as DSM, DTM, Hillshade, Intensity, Number of Returns and PCA, as well as the RGB bands of the Orthographic photographs were used to classify Built-up, Mangroves, Other Trees and Sugarcane. 
In addition, some scholars have carried out coastal vegetation studies using airborne LiDAR, such as the Leaf area index [6], tree height estimation [7], target classification with high resolution multi-spectral imagery $[8,9]$.

Due to the large amount of point cloud data acquired by airborne LiDAR, it is difficult to directly classify and extract the geometric data of point clouds. Aiming at the typical hazard bearing targets such as buildings and trees, this paper presents a method of automatic extraction of disaster bearing targets from coastal LiDAR data, based on geo-referenced feature image of the point clouds.

\section{EXTRACTION OF DISASTER-BEARING TARGETS IN COASTAL ZONE}

\section{A. Preprocessing of Coastal LiDAR data}

The coastal LiDAR point cloud contains some noise data and a large number points of water surface, which should be removed before the extraction of the coastal disaster-bearing targets. The point cloud of water surface has the same elevation characteristics and is distributed in the lowest point in LiDAR point cloud. In this paper, the elevation of the LiDAR point cloud is firstly calculated by histogram to eliminate the noisy points. Then, the average elevation $(H v)$ of water surface points is determined automatically according to the histogram distribution. Finally, given the buffer threshold $(d)$, we can eliminate the point cloud whose elevation is in the range of $(H v \pm d)$, so as to realize the automatic removal of the point cloud on the water surface.

\section{B. Georeferenced Feature Image Generation}

In this paper, the algorithm proposed in [10] is used to generate the georeferenced feature image of the coastal LiDAR data. Specific steps are as follows: (a) Determine the point cloud feature image width $(W)$, height $(H)$ and ground sampling distance $(G S D)$. (b) Determine the characteristic value (Fij) of grid $(i, j)$ according to the weighting formula in [10] using all points (the number is $n i j$ ) in each grid $(i, j)$. (c) Normalize the grid feature value $(F i j)$ to $0 \sim 255$ gray space to generate the georeferenced feature image of the whole scanning area.

\section{Image Segmentation and Contour Extraction}

According to the gray feature of the generated feature image, the gray-level segmentation method based on the maximum between-class variance [11] is used to separate the ground and Non-ground targets. The segmented feature image contains a large number of disaster-bearing targets, such as buildings, trees. Contour extraction and boundary tracking [12] are applied to the segmented binary image to automatically extract the boundary contours of objects such as buildings, trees.

\section{Extraction of Disaster-bearing Targets}

According to the boundary contours of the disasterbearing targets extracted from the segmented image, all points in the contour of each target are extracted using the corresponding relationship between the pixel grid of feature image and the original airborne LiDAR point cloud. In this way, all disaster-bearing targets especially buildings and trees can be automatically extracted from coastal LiDAR data.

\section{EXPERIMENTS AND RESULTS}

\section{A. Experimental Data}

In this paper, LiDAR point cloud of Haidian Island was chosen as the experimental data. The data were collected by Leica's ALS70 equipment by three flight lines, which contained a total of 8.77 million points. Fig. 1 shows the whole airborne LiDAR data of Haidian Island.

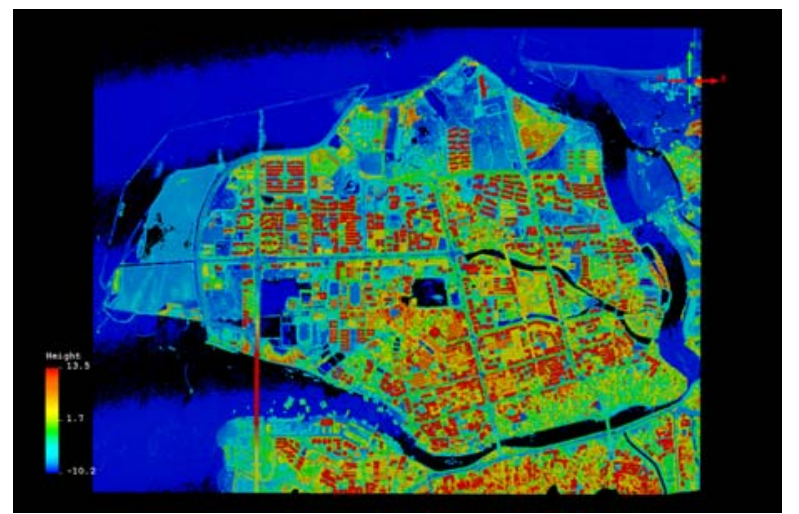

Figure 1. Airborne LiDAR data of Haidian Island.

\section{B. Elimination of The Noise and Water Surface Points}

The characteristics of the coastal LiDAR data were analyzed, such as 3D information, reflection intensity, multiple echo, and so on. The noise and the water surface points were eliminated by the histogram statistics. As shown in Fig.2, 7.95 million points were left.

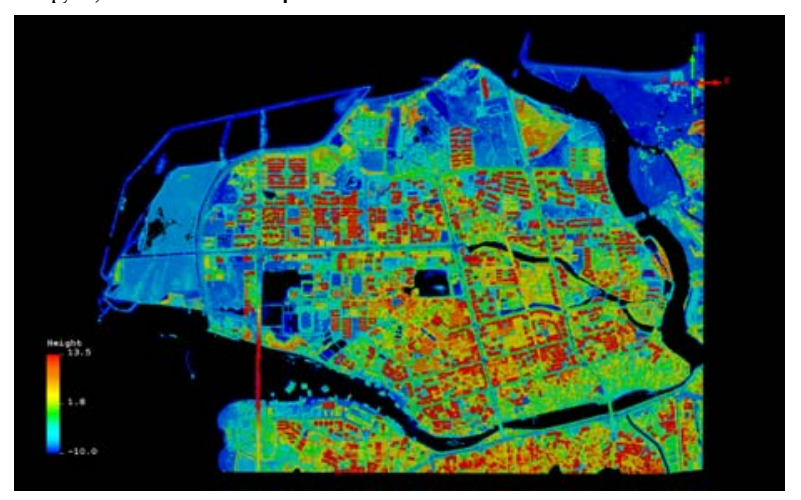

Figure 2. Elimination result of the noise and water surface points.

\section{Generation of Georeferenced Feature Image}

One of the three strips was selected to carry out the extraction experiment of disaster-bearing targets, as shown in Fig.3. Based on this data, the georeferenced feature image of LiDAR point cloud was generated using the proposed algorithm in [10], by setting parameters [10] as $(\alpha=0.2$, $\left.\beta=0.8, C_{c s g}=2.0 \mathrm{~m}\right)$, as shown in Fig. 4 . 


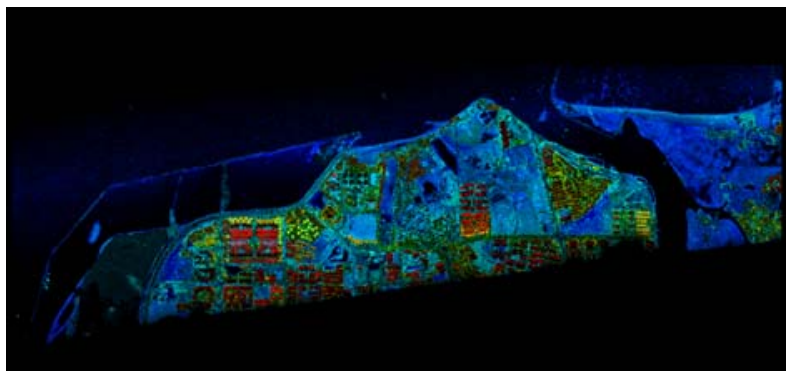

Figure 3. The LiDAR data captured by the first strip.

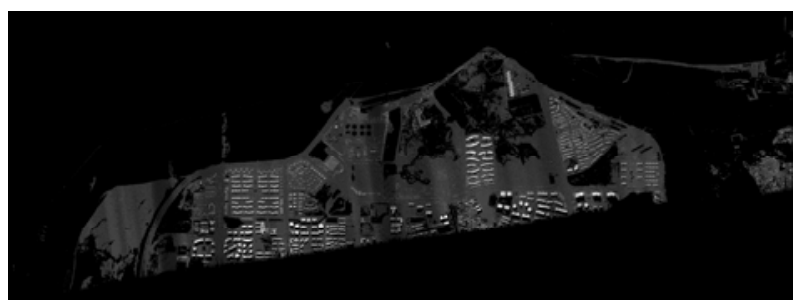

Figure 4. Georeferenced feature image of the first strip LiDAR data.

\section{Extraction of Disaster-bearing Targets}

According to the generated feature image, the disasterbearing targets were separated by image segmentation method, as shown in Fig.5. The contour extraction method was then used to extract the two-dimensional contours of each object in the segmented image, as shown in Fig.6. Combining with the corresponding relationship between the feature image and LiDAR point cloud, the 3D points of each target were extracted according to the two-dimensional contours of targets. Therefore, all the disaster-bearing targets were extracted from the LiDAR point Cloud, as shown in Fig.7.

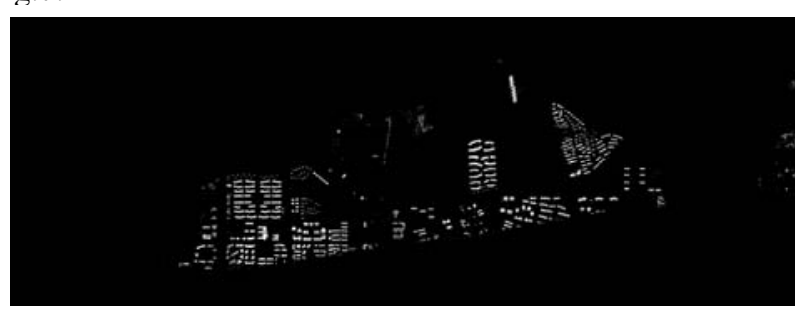

Figure 5. Segmentation of feature image.

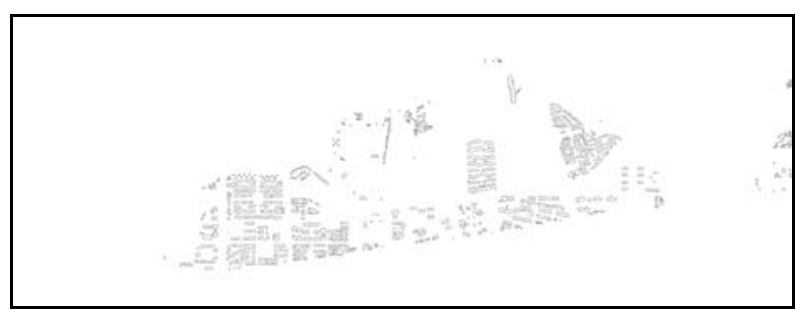

Figure 6. Contour extraction of targets in segmented feature image.

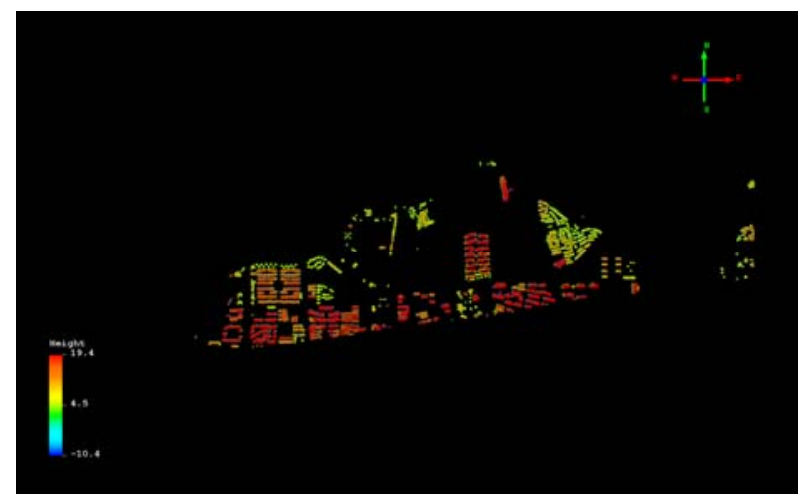

Figure 7. Extracted disaster-bearing targets from coastal LiDAR data.

\section{CONCLUSIONS}

In this paper, the geometric characteristics of the point cloud are used, and the noise and water surface points of the LiDAR point cloud in the coastal zone are automatically eliminated by elevation histogram analysis. Georeferenced feature image is generated for the coastal LiDAR Point cloud, and is applied with methods of image segmentation and contour extraction to extract the typical coastal disasterbearing targets. The results show that the method proposed in this paper transforms the 3D feature of LiDAR point cloud into two-dimensional space, and uses the correspondence between $3 \mathrm{D}$ point cloud and feature image to realize the automatic extraction of disaster-bearing targets, such as buildings and trees. Further research will be carried out to improve the accuracy of target extraction.

\section{ACKNOWLEDGMENT}

Work described in this paper was jointly supported by Public Science and Technology Research Funds Projects of Ocean (No. 201305020-7), National Key Research And Development Program (No. 2016ysc1403200), National Natural Science Foundation of China (No. 61527810), the project of Key Laboratory of Technology for Safeguarding of Maritime Rights and Interests and Application, SOA. Thanks to South China Sea Branch of State Oceanic Administration for providing the datasets.

\section{REFERENCES}

[1] J.C. Brock and S.J. Purkis, "The Emerging Role of Lidar Remote Sensing in Coastal Research and Resource Management,” Journal of Coastal Research, vol. 25(6), 2015, pp.1-5.

[2] K. Pye and S.J. Blott, "Assessment of beach and dune erosion and accretion using LiDAR: Impact of the stormy 2013-14 winter and longer term trends on the Sefton Coast, UK,”. Geomorphology, vol. 266, 2016, pp.146-167.

[3] H. Liu, D. Sherman, and S. Gu, "Automated Extraction of Shorelines from Airborne Light Detection and Ranging Data and Accuracy Assessment Based on Monte Carlo Simulation,” Journal of Coastal Research, vol. 23(6), 2016, pp.1359-1369.

[4] X. Zhang and C. Glennie. "Adolication of LiDAR Data for Monitoring Coastal Change in Galveston,” $\mathrm{THC}-2012$ Conference \& Exhibition. 
[5] A.V. Pada, J. Silapan, M.A. Cabanlit, F. Campomanes, and J.J. Garcia, "Mangrove Forest Cover Extraction of the Coastal Areas of Negros Occidental, Western Visayas, Philippines Using LIDAR Data," International Archives of the Photogrammetry, Remote Sensing \& Spatial Information Sciences, vol.XLI-B1, 2016, pp.73-79.

[6] D.vRiano, F. Valladares, S. Condés, and E. Chuvieco, "Estimation of leaf area index and covered ground from airborne laser scanner (LiDAR) in two contrasting forests," Agricultural and Forest Meteorology, Vol. 124, 2004, pp. 269275.

[7] S.C. Popescu, R.H. Wynne, and R.F. Nelson, "Estimating plotlevel tree heights with LiDAR: local filtering with a canopyheight based variable window size," Computers and Electronics in Agriculture, vol. 37, 2002, pp. 71-95.
[8] D.S. Lee and J. Shan, "Combining LiDAR elevation data and IKONOS multispectral imagery for coastal classification mapping,” Marine Geodesy, vol. 26, 2003, pp. 117-127.

[9] P. Kempeneers, B. Deronde, S. Provoost, and R. Houthuys, "Synergy of Airborne Digital Camera and LiDAR Data to Map Coastal Dune Vegetation,” Journal of Coastal Research, vol. 53, 2009, pp. 73-82.

[10] B.S. Yang, Z. Wei, Q.Q. Li, and J. Li, "Automated extraction of street-scene objects from mobile lidar point clouds," International Journal of Remote Sensing, vol.33(18), 2012, pp. 5839-5861.

[11] N. Ostu, "A Threshold Selection Method from Gray Level Histogram,” IEEE Trans System Man Cement, vol.SMC-9, 1979, pp. 62-66.

[12] T. Pavlidis, Algorithms for Graphics and Image Processing, Maryland: Computer Science Press, 1982. 